\title{
Suppression of the Generator Imposition Vibration
}

\author{
Evžen Thöndel and Jiř́ Vondřich \\ Czech Technical University in Prague, Faculty of Electrical Engineering, Department of Electric Drives and Traction, \\ Prague, Czech Republic, \\ e-mail: thondee@fel.cvut.cz,vondrich@fel.cvut.cz
}

\begin{abstract}
Generator vibrations occur as a result of unbalanced revolving masses, loading forces and torques, starting and coasting of driving motors and other effects. Suitable mounting of the generator is possible using damping components such as steel springs, pneumatic springs, rubber pads or other damping components. The article describes a numerical calculation of individual deviations of the generator in dependence on time for the given machine parameters and the selected damping components of its mounting using the Matlab program.
\end{abstract}

Keywords - generator, vibration, numerical calculation, Matlab

\section{INTRODUCTION}

It is possible to prevent undesirable vibrations of machines and their bearing and non-bearing structures by a suitable mounting of the machine to the base. Synchronous generators used for conversion of mechanical energy to electric energy represent one group of the machines requiring assessment from the viewpoint of dynamic effects on structures and bases. The following aspects belong among the principal ones for a reliable design of structures or bases under the machinery inducing dynamic effects:

- Effective rate of response of the oscillation speed in $\mathrm{mm} / \mathrm{s}$ to the frequency of operational speed and potentially also in transient conditions at a machine starting or coasting when occurrence of resonance peaks of the monitored parameter response takes place as a result of excitation of significant own frequencies and oscillation shapes.

- Admissible level of vibration of the base in the place of equipment mounting sensible to the level of mechanical vibrations (such as laboratory scales, spectrometers, etc.) the level of the limit admissible amplitudes of offsets required by the manufacturer ranges in orders of units up to hundredths of $\mu \mathrm{m}$.

- Limit values of the oscillation response at which it is possible to assume occurrence of visible defects on building bearing and non-bearing structures - limit acceleration amplitudes in $\mathrm{mm} / \mathrm{s}^{2}$ for dynamic effects with frequencies up to $10 \mathrm{~Hz}$ and oscillation amplitude in $\mathrm{mm} / \mathrm{s}$ for effects with frequencies within the range from 10 to $100 \mathrm{~Hz}$ for the occasional impact loading and stabilized periodic oscillation are specified.

- Base frames on which machinery is mounted should be in resonance with the operational rotation speed of any machine part (e.g. of a generator with the speed frequency of $25 \mathrm{~Hz}$ and turbine with frequency of $50 \mathrm{~Hz}$ ) - it is understood usually by this requirement that the dominant own frequency (i.e. e.g. basic frequency of the bending oscillation of a ceiling component or frequency of the cross oscillation of the turbo-bench frame) should differ by at least $\pm 20 \%$ from the frequency of the operational rotation speed; it is recommended in the case of ceiling structures that their first bending own frequency at longterm and so also the most frequent operational loading should be at least $4 \mathrm{~Hz}$ - this requirement guarantees elimination of resonance oscillation of ceilings when walking and can be ensured usually by a sufficient bending rigidity of the bearing structure.

- Amplitudes of the response of internal forces or stress in the structure ascertained by a dynamic calculation when maximum dynamic load is considered - in case of rotational machines, maximum unbalanced masses on the revolving parts of the machine arising in the case of rotor failure (such as turbine blade braking); when assessing the structure according to limit conditions of the load bearing capacity, it is necessary to take into consideration amplitudes of the monitored response parameter (such as bending torque in cross section) within the complete frequency spectrum, i.e. not only at the operational speed but also in transient conditions, i.e. also in resonance peaks or in the complete time section of the acting load (such as short-circuit torque).

- Assessment of the structure for a highly cyclic fatigue stress - it should be carried out in the cases when the stress amplitude or internal forces in the assessed cross sections of the structure at the machine operational speed is significant when compared with the effect of static load acting in a long-term period or if the dynamic effect causes an oscillation of stress; a risk of occurrence of a brittle fracture in case of welded steel structures but also fatigue damage of ferroconcrete structures are characteristic examples.

Vibrations take place in generators when passing through critical speed and also because of drying of the insulation material in slots, formation of backlash and potentially as a result of other unexpected effects. We distinguish between high-speed generators (turbogenerators) driven by steam or gas turbines and low-speed generators driven by water turbines. Turbo-generators usually have a small diameter of the revolving parts (because of a reduction of centrifugal forces), long axial length and horizontal position. Two- and four-pole machines are used typically (in case of generators for the $50 \mathrm{~Hz}$ networks, speed is either 3,000 rpm or 1,500 rpm). 
The low-speed generators usually have the speed of $500 \mathrm{rpm}$ and lower, corresponding to number of poles, larger diameter and shorter axial length. All types have movable and static parts (rotor and stator manufactured of a magnetic material.

The stator winding that powers the system is positioned in slots distributed equidistantly along its internal periphery and consists of three identical parts pertaining to individual phases. The direct current excitation winding of turbo-generators is positioned in a similar way in the slots on the rotor while in the case of low-speed machines, on their salient poles.

Moreover, the rotor is provided with the damping winding (amortisseur) the task of which is to damp the rotor mechanical oscillation. This winding is formed by conductive wedges in the slots of the excitation winding in turbo-generators while in the case of the low-speed generators it is positioned in the pole axial slots.

Rotor direct excitation current induces revolving magnetic field in the machine the intensity of which is proportional to this current. The created magnetic flux then induces an electro-motoric force in each of three phases of the stator winding and as a result of it a current arises and corresponding power will start to flow in the system.

The current flowing through the stator winding creates its own magnetic field that has a constant magnitude; however, it is revolving at the same speed like the rotor. The corresponding magnetic flux is superposed on the flux induced by the excitation winding. The arisen resulting flux has a stationary character with respect to the rotor; however, it revolves at a constant speed with respect to the stator.

The consequence is that the rotor can be massive (eddy currents are not induced in it so that corresponding losses do not also occur here) while the stator is laminated because of opposite reasons. If the speed of the rotor deviates from the synchronous speed for any reason, the resulting flux with respect to the rotor will not have the stationary character, which will result in formation of currents in particular in the damping winding. These currents will induce flux in the opposite direction according to the Lenz rule and prevent a change of the synchronous speed (and thus hunting).

Universal trend to increasing the nominal power of the new constructed generators and power plants existed in former years as relative investment and operational costs are reduced with growing power of the units (lower specific weight of the generator for unit power, smaller buildings, general built-up area and altogether all required equipment in the same sense). However, the opposite tendency has appeared in some countries recently, in particular where cheap natural gas is available. Power plants with gas turbines with a combined cycle driving generators up to power of $250 \mathrm{MVA}$ are produced. However, modern synchronous generators with power of $100-1300 \mathrm{MW}$ and voltage of $10-32 \mathrm{kV}$ are used commonly. In case of turbo-generator, we assume formation of vibrations as a result of unbalance of the rotor.

We will describe the model with motion equations in a matrix representation. After a modification, it is possible to determine the transfer function $G(p)$ from which it is then possible to determine the courses of the amplitude frequency response curves for individual degrees of freedom using the Matlab program. The courses of the speed in dependence on time for individual degrees of freedom will be determined numerically then from the motion equations for the given stabilized generator displacements.

\section{GENERATOR MODEL}

Fig. 1 illustrates a simplified model of a three-phase two-pole generator (type 4A 275-02H, power $35 \mathrm{MVA}$, $\mathrm{n}=3000 \mathrm{rpm}$, product of ČKD Nové Energo a.s.) in which vibrations are caused as a consequence of an unbalance $U$ of the revolving part given by the equation:

$$
U=m_{n} e,
$$

where $m_{n}$ is mass of the revolving part and $e$ is eccentricity. The generator is intended for connection with a steam turbine. The complete original set was firmly fixed to a concrete area. Because the complete set except the generator remained firmly fixed to the concrete area, a flexible mounting of a newly installed generator was required. Requirement of a numerical design of damping components in the case of rotor unbalance that was detected in the former generator also appeared.

In order to damp vibrations, the generator will be mounted on 8 damping components in the direction of $x, y$ and $z$ axes as indicated in Fig. 1. The damping components are represented by sets of steel springs (Gerb) with known constants of rigidity $k_{x}, k_{y}, k_{z}$, damping constant $b_{x}=b_{y}=b_{z}$ of the components in the directions of $x, y$ and $z$. The generator model represents a mechanical system with six degrees of freedom $\mathrm{x}, \mathrm{y}, \mathrm{z}, \varphi_{\mathrm{x}}, \varphi_{y}, \varphi_{z}$.

The system can be described by a motion equation in the matrix representation

$$
m \ddot{\bar{x}}=k \bar{x}+b \dot{\bar{x}}+F,
$$

where

$$
m=\left[\begin{array}{cccccc}
m & 0 & 0 & 0 & 0 & 0 \\
0 & m & 0 & 0 & 0 & 0 \\
0 & 0 & m & 0 & 0 & 0 \\
0 & 0 & 0 & I_{x} & 0 & 0 \\
0 & 0 & 0 & 0 & I_{y} & 0 \\
0 & 0 & 0 & 0 & 0 & I_{z}
\end{array}\right], \quad x=\left[\begin{array}{c}
x \\
y \\
z \\
\varphi_{x} \\
\varphi_{y} \\
\varphi_{z}
\end{array}\right], \quad F=\left[\begin{array}{c}
m_{n} e \Omega^{2} \cos \Omega t \\
0 \\
m_{n} e \Omega^{2} \sin \Omega t \\
m_{n} e \Delta y \Omega^{2} \sin \Omega t \\
0 \\
-m_{n} e \Delta y \Omega^{2} \cos \Omega t
\end{array}\right]
$$


$k=\left[\begin{array}{cccccc}-8 k_{x} & 0 & 0 & 0 & 8 k_{x} h & 0 \\ 0 & -8 k_{y} & 0 & -8 k_{y} h & 0 & 0 \\ 0 & 0 & -8 k_{z} & 0 & 0 & 0 \\ 0 & -8 k_{y} h & 0 & -8\left(\frac{v^{2}}{4} k_{z}+k_{y} h^{2}\right) & 0 & 0 \\ 8 k_{x} h & 0 & 0 & 0 & -8\left(k_{z} a^{2}+k_{x} h^{2}\right) & 0 \\ 0 & 0 & 0 & 0 & 0 & -8\left(k_{x} \frac{v^{2}}{4}+k_{y} a^{2}\right)\end{array}\right]$

$$
b=\left[\begin{array}{cccccc}
-8 b & 0 & 0 & 0 & 168 h & 0 \\
0 & -8 b & 0 & -8 b h & 0 & 0 \\
0 & 0 & -8 b & 0 & 0 & 0 \\
0 & -8 b h & 0 & -8 b\left(\frac{v^{2}}{4}+h^{2}\right) & 0 & 0 \\
8 b h & 0 & 0 & 0 & -8 b\left(a^{2}+h^{2}\right) & 0 \\
0 & 0 & 0 & 0 & 0 & -8 b\left(\frac{v^{2}}{4}+a^{2}\right)
\end{array}\right] \text {, }
$$
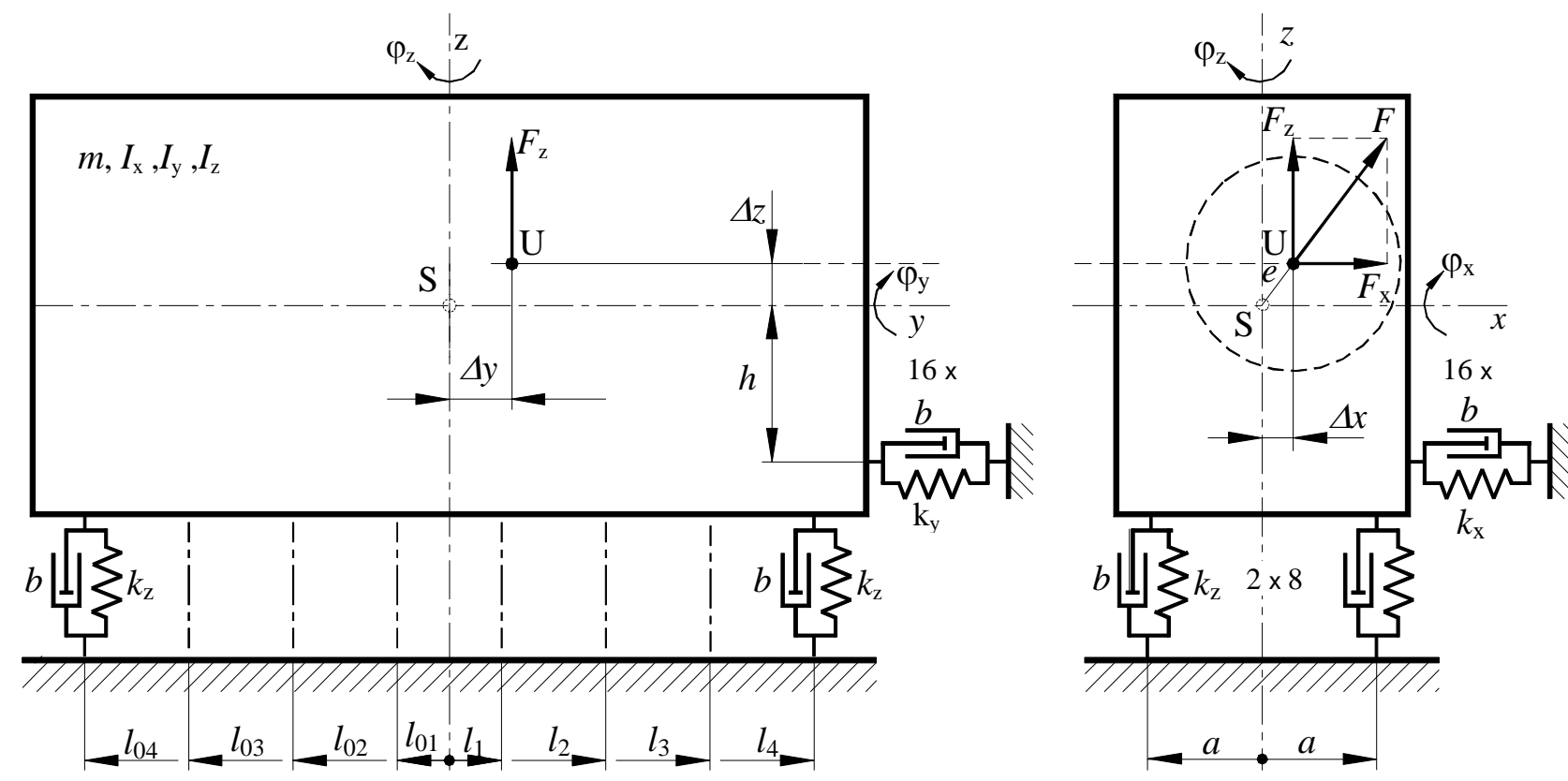

Fig. 1 Generator model

where

$$
v^{2}=l_{1}^{2}+l_{2}^{2}+l_{3}^{2}+l_{4}^{2}+l_{01}^{2}+l_{02}^{2}+l_{03}^{2}+l_{04}^{2} .
$$

If we carry out a modification of Eq. (6)

If we introduce

$$
\dot{\bar{x}}_{1}=m^{-1} b \bar{x}_{1}+m^{-1} k \bar{x}_{2}+F, \quad \dot{\bar{x}}_{2}=\bar{x}_{1},
$$

$$
\dot{\bar{x}}=\bar{x}_{1}, \quad \bar{x}=\bar{x}_{2},
$$

it is possible to rewrite the motion equation in the form

Eq. (2) can be developed in the form

$$
\dot{\hat{x}}=A \bar{x}+B F, \quad \hat{y}=C \hat{x},
$$

$$
m \dot{\bar{x}}_{1}=b \bar{x}_{1}+k \bar{x}_{2}+F, \quad \dot{\bar{x}}_{2}=\bar{x}_{1,}
$$

(6) where 


$$
\begin{gathered}
A=\left[\begin{array}{cc}
m^{-1} b & m^{-1} k \\
I_{6 x 6} & 0_{6 x 6}
\end{array}\right]_{12 \times 12} \quad \hat{x}=\left[\begin{array}{c}
\hat{x}_{1} \\
\hat{x}_{2}
\end{array}\right]_{12 x 1} \quad B=\left[\begin{array}{c}
m_{6 x 6}^{-1} \\
0_{6 x 6}
\end{array}\right]_{12 x 6} \\
\hat{y}=\left[\begin{array}{c}
x \\
y \\
z \\
\varphi_{x} \\
\varphi_{y} \\
\varphi_{z}
\end{array}\right] \quad C=\left[\begin{array}{cccccccccccc}
0 & 0 & 0 & 0 & 0 & 0 & 1 & 0 & 0 & 0 & 0 & 0 \\
0 & 0 & 0 & 0 & 0 & 0 & 0 & 1 & 0 & 0 & 0 & 0 \\
0 & 0 & 0 & 0 & 0 & 0 & 0 & 0 & 1 & 0 & 0 & 0 \\
0 & 0 & 0 & 0 & 0 & 0 & 0 & 0 & 0 & 1 & 0 & 0 \\
0 & 0 & 0 & 0 & 0 & 0 & 0 & 0 & 0 & 0 & 1 & 0 \\
0 & 0 & 0 & 0 & 0 & 0 & 0 & 0 & 0 & 0 & 0 & 1
\end{array}\right]_{6 \times 12}
\end{gathered}
$$

The transfer function $G(p)$ can be determined from equations

$$
p \widehat{x}=A \hat{x}+B F, \quad \hat{y}=C \hat{x} .
$$

From Eqs. (10), it is then possible to determine

$$
\widehat{y}=C(p I-A)^{-1} B F,
$$

where the transfer function can be expressed as

$$
G(p)=C(p I-A)^{-1} \text {. }
$$

Using the transfer function, it is then possible to determine the courses of the resonance curves for individual degrees of freedom $x, y, z$ by means of the Matlab program.

\section{RESULTS OF THE SOLUTIONS}

For the given values of the rigidity constants $k_{x}, k_{y}, k_{z}$ and damping constants $b=b_{x}=b_{y}=b_{z}$ of the individual damping components and the given value of mass $m$ and generator moments of inertia $I_{x}, I_{y}, I_{z}$ (Fig. 1), we look for the amplitude frequency response curves (Fig. 3) using numeric calculation method with the Matlab-Simulink

program (Fig. 2) by means of the transfer function. We also solve the courses of displacements $\mathrm{x}, \mathrm{y}, \mathrm{z}, \varphi_{\mathrm{x}}, \varphi_{y}, \varphi_{z}$. in dependence on time (Fig.4) using the numeric calculation. The parameters of the generator, its mounting and damping components are:

$$
\begin{aligned}
& m=40430 \mathrm{~kg}, \quad m_{n}=14210 \mathrm{~kg}, I_{x}=10 \mathrm{kgm}^{2}, I_{y}=947 \mathrm{kgm}^{2}, \quad I_{z}=10 \mathrm{kgm}^{2}, \\
& l_{1}=317,5 \mathrm{~mm}, \quad l_{4}=952,5 \mathrm{~mm}, l_{01}=317,5 \mathrm{~mm}, l_{04}=952,5 \mathrm{~mm}, \quad l_{2}=l_{3}=l_{02}=l_{03}=0 \text {, } \\
& a=1,17 \mathrm{~m}, \quad h=0,497 \mathrm{~m}, \quad \Delta y=0 \mathrm{~mm}, \quad n=3000 \mathrm{~min}^{-1}, \Omega=\pi n / 30, \\
& k_{x}=k_{y}=1,5 \cdot 10^{5} \mathrm{Nm}^{-1}, \quad k_{z}=3 \cdot 10^{5} \mathrm{Nm}^{-1}, \quad b=2000 \mathrm{Nsm}^{-1} \text {. }
\end{aligned}
$$

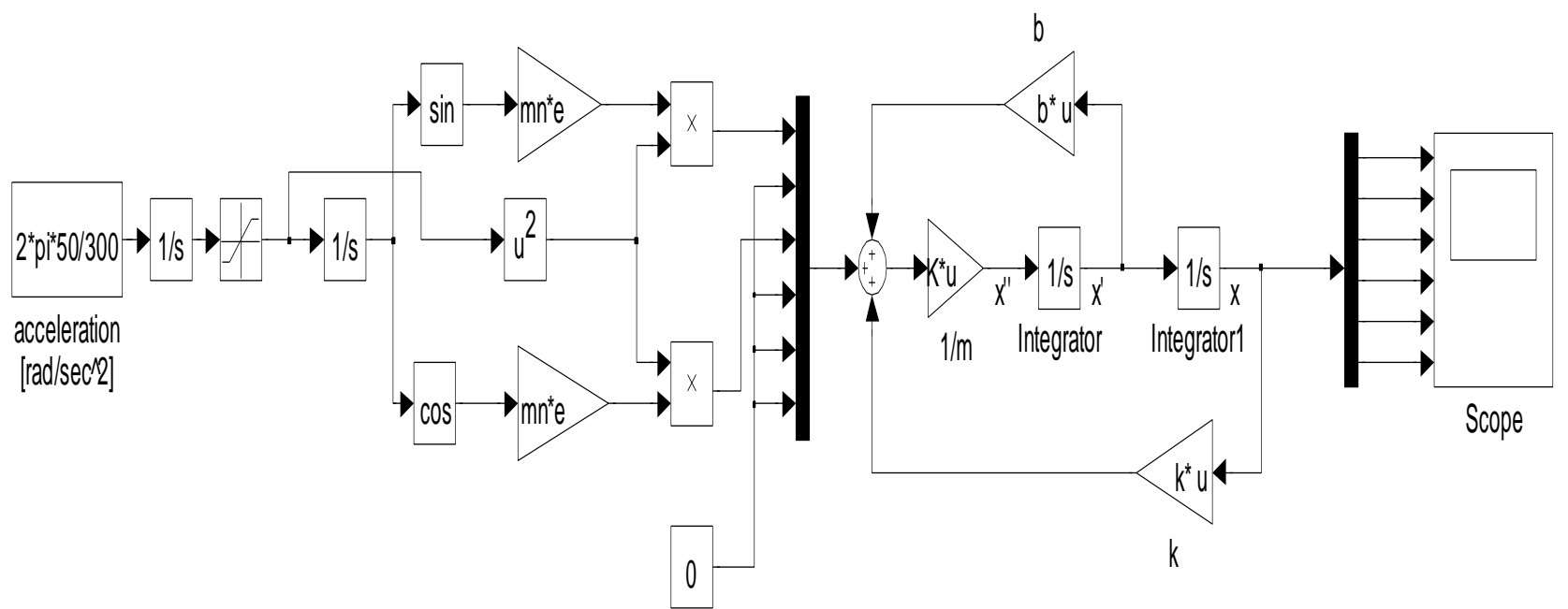

Fig. 2 Simulink scheme. 


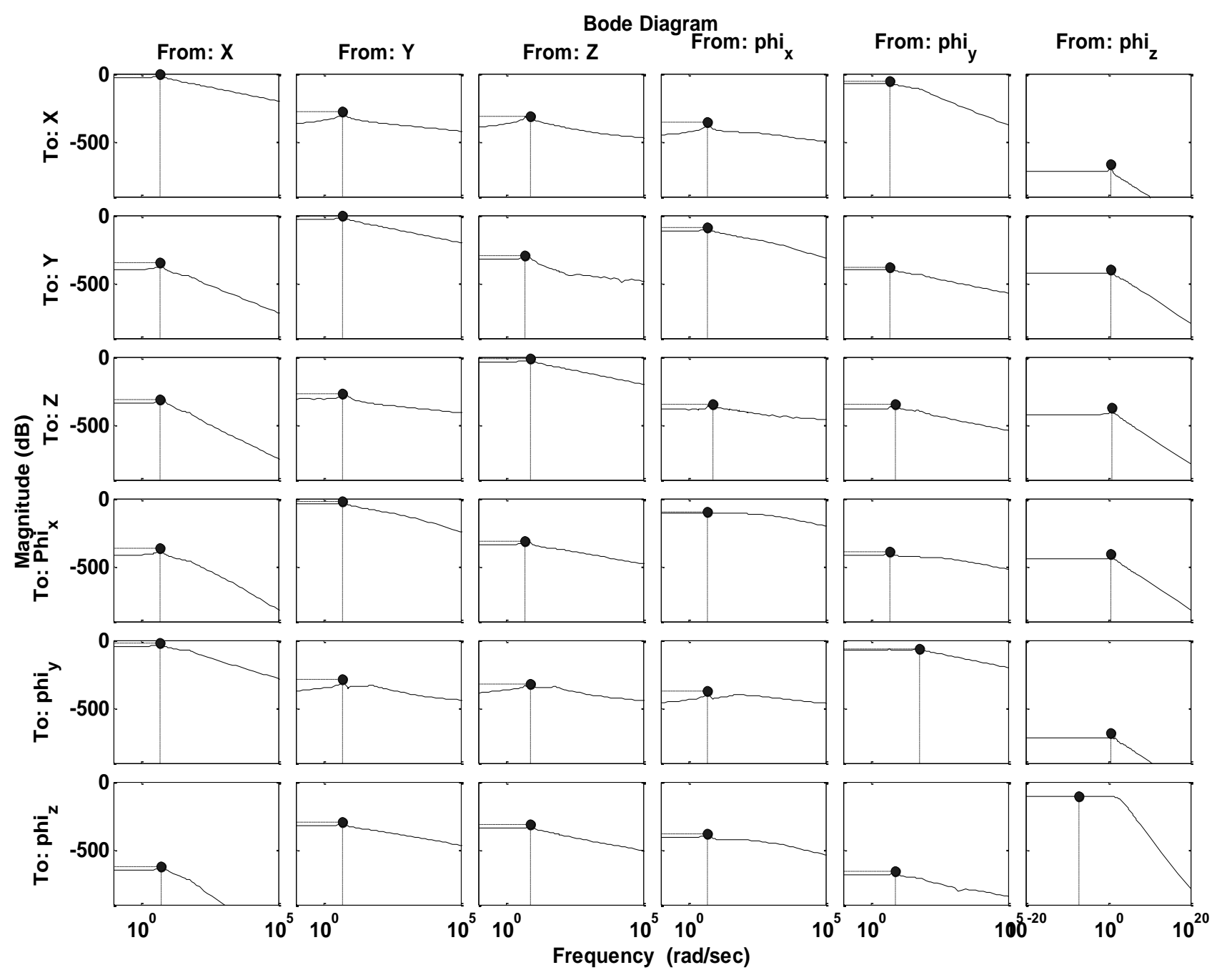

Fig. 3 Amplitude frequency response.

\section{CONCLUSION}

In the case that it is possible to describe the motion of a vibrating machine model by a system of linear differential equations, it is then possible to express the transfer function $G(p)(12)$ and the courses of the amplitude frequency response curves (Fig. 3) from which it is apparent that maximum displacements by three resonant frequencies $\omega_{1}=5,14 \mathrm{~s}^{-1}\left(x_{\max }=5,5 \mathrm{~mm}\right.$, $\left.\varphi_{\text {ymax }}=0,9510^{-3} \mathrm{rad}\right), \omega_{2}=7,7 \mathrm{~s}^{-1}\left(z_{\max }=8,5 \mathrm{~mm}\right)$, $\omega_{3}=58,14 \mathrm{~s}^{-1}\left(\varphi_{y \max 2}=0,6110^{-3} \mathrm{rad}\right)$ (Fig. 4). Word "data" is plural, not singular. The courses of deviations $\mathrm{x}, \mathrm{y}, \mathrm{z}, \varphi_{\mathrm{x}}, \varphi_{y}, \varphi_{z}$. in dependence on time (Fig. 4) at the operational speed of the turbo-generator $n=3,000 \mathrm{rpm}$ were determined numerically using the Matlab-Simulink program: $x=1,1 \mathrm{~mm}, z=1,2 \mathrm{~mm}$, $\varphi_{y}=0,210^{-3} \mathrm{rad}$ (Fig. 2).
To reduce the vibrations considerably, it is suitable for a new set to mount it flexibly to a board that is mounted flexibly to a rigid base. The board will then act as an absorber accepting energy of the set oscillations. It is then possible to determine the mass of the board and the constants of rigidity of the damping components using numerical calculation.

\section{REFERENCES}

[1] Using Simulink, version 5.The Math Works, Inc., Natic. July 2002, 484 pgs. 

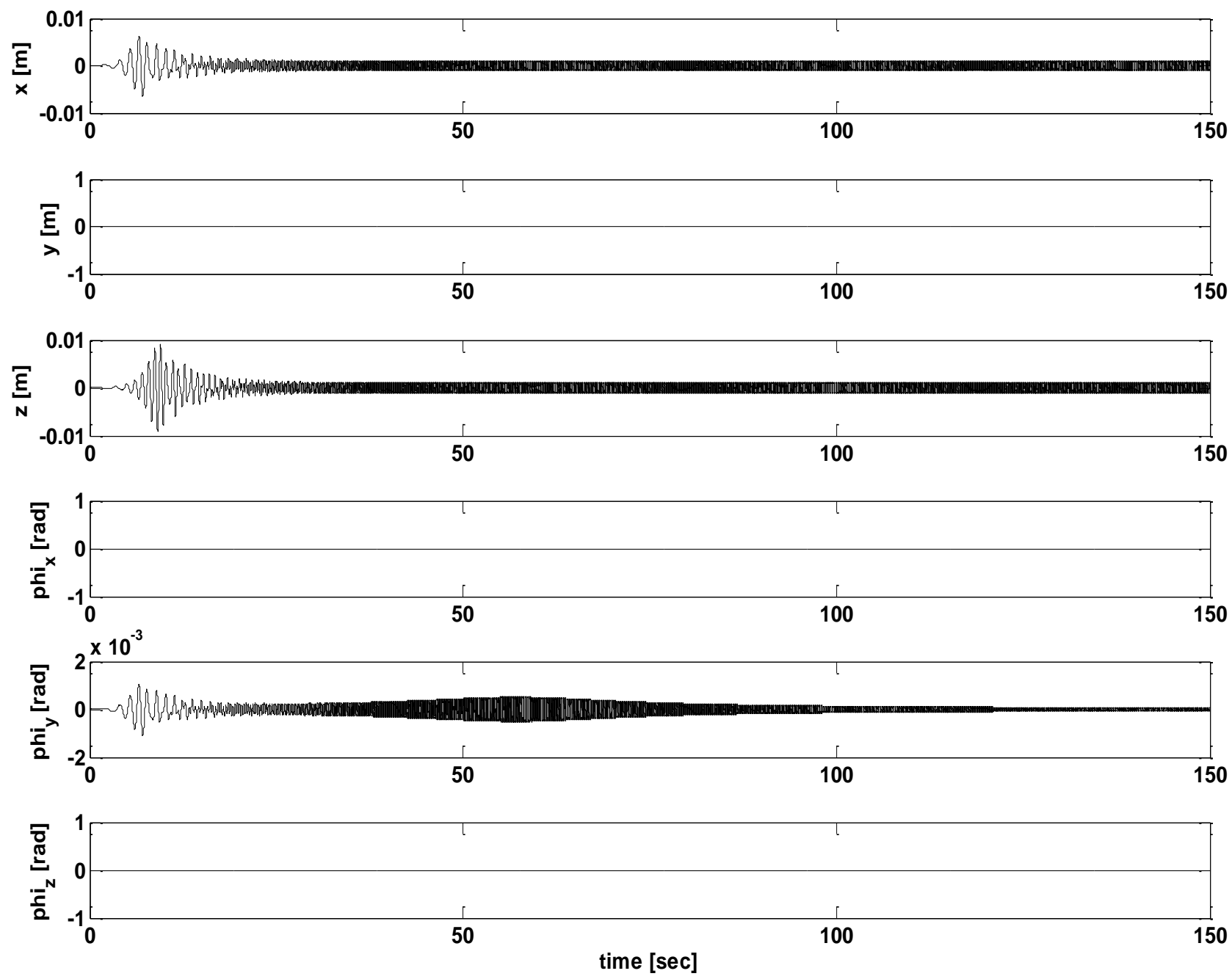

Fig. 4 Deviations $\mathrm{x}, \mathrm{y}, \mathrm{z}, \varphi_{\mathrm{x}}, \varphi_{y}, \varphi_{z}$. of the generator. 